\title{
Computational study of structural changes in neuronal networks during growth: a model of dissociated neocortical cultures
}

\author{
Jugoslava Aćimović ${ }^{*}$, Tuomo Mäki-Marttunen ${ }^{1,2}$, Marja-Leena Linne ${ }^{1}$ \\ From Twentieth Annual Computational Neuroscience Meeting: CNS*2011 \\ Stockholm, Sweden. 23-28 July 2011
}

Networks of neurons possess distinct structural organization that constraints generated activity patterns, and consequently, the functions of the system. The emergence of the network structure can be understood by studying the rules that govern growth of neurons and their self-organization into neuronal circuits. We analyze these rules using a computational model of growth developed for dissociated neocortical cultures. Compared to the growth in vivo, the cultures represent simplified two dimensional systems that still possess the intrinsic properties of single neurons although they lack the natural extracellular environment present in vivo. This setup provides a possibility to address in depth the selected mechanisms that affect neuronal growth. The collected structural data (through staining and microscopy) and electrophysiological data (using microelectrode arrays) facilitate validation of computational models. Neuronal growth in dissociated cultures has been examined in several studies in order to access the role of activity in network development [6],[7] or to extract the structural changes during growth from the recorded activity and identify the significant time points in network development [4]. In addition, two simulators of neuronal growth were recently published to aid the development of computational models [3],[9]. Their performance, in context of modeling neocortical cultures, is compared in [1].

The analyzed model consists of two types on neurons, most commonly observed in the neocortical cultures, the pyramidal cells and the nonpyramidal GABAergic cells, placed in a dish-like space with the density of cells

\footnotetext{
* Correspondence: jugoslava.acimovic@tut.fi

'Department of Signal Processing, Tampere University of Technology,

Tampere, Finland

Full list of author information is available at the end of the article
}

corresponding to the experimental values. The phenomenological model that takes into account growth of every neurite is constructed using the description from the literature [3],[8]. It is compared to the model that defines only the overall shape of each neuritic field. We examine the critical time point in network development, i.e. the emergence of fully connected networks [2],[4], which is dependent on the overall growth speed of neurites. The local structural features are accessed using the frequency of motifs in networks [2],[5]. Local connectivity patterns, captured by the motif counts, depend on the shape of neurites and distribution of synaptic contacts along neurites. The goal of this study is to analyze model dynamics through evaluation of the proposed measures. The dependence on model parameters is examined in details, particularly, whether small variations in parameter values significantly affect both measures of network structure. The obtained conclusions are compared to the experimental findings from the literature $[4,5]$.

\section{Acknowledgements \\ The authors would like to acknowledge the following funding: Academy of Finland project no. 213462 (Center of Excellence in Signal Processing), and} TISE graduate school.

\section{Author details}

${ }^{1}$ Department of Signal Processing, Tampere University of Technology, Tampere, Finland. ${ }^{2}$ Department of Mathematics, Tampere University of Technology, Tampere, Finland.

Published: 18 July 2011

\section{References \\ 1. Aćimović J, Mäki-Marttunen T, Havela R, Teppola H, Linne ML: Models of neuronal growth in vitro: Comparison of two simulators of growth, CX3D and NETMORPH. EURASIPJournal Bioinf Syst Biol .}

\section{( Biomed Central}


2. Dorogovtsev S, Mendez J: Evolution of Networks: From Biological Nets to the Internet and WWW. Oxford University Press, NT; 2003.

3. Koene RA, Tijms B, van Hees P, Postma F, de Ridder A, Ramakers GJA, van Pelt J, van Ooyen A: NETMORPH: A Framework for the Stochastic Generation of Large Scale Neuronal Networks With Realistic Neuron Morphologies. Neuroinformatics 2009, 7(3):195-210.

4. Soriano J, Martinez MR, Tlusty T, Moses E: Development of input connections in neural cultures. Proc Natl Acad Sci U S A 2008, 105(37):13758-13763.

5. Sporns O: Networks of the brain. The MIT Press, Cambridge, Massachusetts; 2011.

6. Tetzlaff C, Okujeni S, Egert U, Wörgötter F, Butz M: Self-Organized Criticality in Developing Neuronal Networks. PLoS Comp Biol 2010, 6(12).

7. van Ooyen A, van Pelt J, Corner MA, Kater SB: Activity-Dependent Neurite Outgrowth: Implications for Network Development and Neuronal Morphology. Modeling Neural Development The MIT Press, Cambridge, Massachusetts; 2003.

8. van Pelt J, Uylings HBM: Natural Variablity in the Geometry of the Dendritic Branching Patterns. Modeling in the Neurosciences: From Biological Systems to Neuromimetic Robotics Taylor\&Francis, Boca Raton, Florida, USA; 2005

9. Zubler F, Douglas R: A framework for modeling the growth and development of neurons and networks. Frontiers Comp Neurosci 2009, $3(25)$.

doi:10.1186/1471-2202-12-S1-P203

Cite this article as: Aćimović et al: Computational study of structural changes in neuronal networks during growth: a model of dissociated neocortical cultures. BMC Neuroscience 2011 12(Suppl 1):P203.

\section{Submit your next manuscript to BioMed Central and take full advantage of:}

- Convenient online submission

- Thorough peer review

- No space constraints or color figure charges

- Immediate publication on acceptance

- Inclusion in PubMed, CAS, Scopus and Google Scholar

- Research which is freely available for redistribution

Submit your manuscript at www.biomedcentral.com/submit 